\title{
ANÁLISIS DE LA ATENUACIÓN EN MATERIALES DIDÁCTICOS DE ELE DE NIVEL B2 A LA LUZ DEL PCIC
}

\author{
AN ANALYSIS OF MITIGATION IN SFL B2 LEVEL DIDACTIC MATERIALS \\ ACCORDING TO THE PCIC
}

\author{
Alba Negre Parra \\ Universistat de València \\ albanegre93@gmail.com
}

Recibido: 20/12/2016

Aceptado: 06/04/2017

\section{Resumen}

Partiendo del estudio teórico de la atenuación se ha llegado al análisis contrastivo de siete manuales de ELE. El principal objetivo ha sido el análisis de los manuales para determinar el tratamiento que le conciernen a la atenuación, así como su adaptación a las directrices del Plan Curricular del Instituto Cervantes. Se han extraído los resultados de los manuales para comparar el tratamiento que le dan según el enfoque didáctico que sigue el manual. Después, se ha comparado la presencia y/o ausencia de los mecanismos de atenuación según lo establecido por el PCIC. Una vez hecho el análisis y valorados los resultados, se ha llegado a la conclusión de que no existe el manual perfecto.

PALABRAS CLAVE: atenuación pragmática, ELE, análisis de manuales, nivel de lengua $\mathrm{B} 2$, manuales.

\begin{abstract}
This is a contrastive analysis of seven manuals of SFL (Spanish as a Foreign Language) based on the theoretical study of mitigation. The main objective is the analysis of the manuals in order to determine how mitigation is taken into account, as well as its adaptation to the guidelines of Curriculum Plan of the Instituto Cervantes. The results of each manual have been extracted to compare the treatment according to the didactic approach of the manual. After, I have compared the presence/absence of mitigation strategies as established by the PCIC. Once the analysis has been done and the results have been evaluated, it has been concluded that the perfect manual does not exist.
\end{abstract}

KEY WORDS: pragmatic mitigation, SFL, manual analysis, B2 language level, manuals.

Para citar este artículo / To cite this article: Negre Parra, Alba (2018): Análisis de la atenuación en materiales didácticos de ELE de nivel B2 a la luz del PCIC. García Ramón, Amparo y Soler Bonafont, María Amparo (Eds.): ELUA: Estudios de atenuación en el discurso, Anexo IV, págs. 269-288.

Enlace / Link: http://dx.doi.org/10.14198/ELUA2018.Anexo4.15 


\section{INTRODUCCIÓN}

El presente artículo se centra en la atenuación, fenómeno poco conocido y trabajado en el ámbito de la enseñanza, y más concretamente, en el caso del español como lengua extranjera (en adelante, ELE). La enseñanza de la atenuación en ELE está ligada al desarrollo de la competencia comunicativa y de la competencia pragmática.

Este es un estudio empírico, cuyos dos objetivos generales son, en primer lugar, realizar un estudio crítico sobre el tratamiento de la atenuación en los manuales comerciales de ELE de nivel B2 y destacar de esta manera las carencias pragmáticas y didácticas de este fenómeno. En segundo lugar, se ha realizado un análisis del fenómeno para observar en qué medida se adaptan a las directrices del Plan Curricular del Instituto Cervantes (PCIC, Instituto Cervantes 2006). Hasta hoy apenas se ha abordado en la bibliografía la necesidad de enseñar fenómenos pragmáticos en la didáctica de ELE, por lo que con esta investigación se pretende contribuir a cubrir un hueco en este ámbito, a través del estudio de un fenómeno concreto como es la atenuación.

De este objetivo general se derivan otros objetivos particulares: el análisis comparativo de los distintos manuales según su enfoque didáctico (enfoque comunicativo, por tareas, orientado a la acción); el análisis comparativo según la finalidad de la enseñanza (español para fines generales o español para fines específicos); y, posteriormente, el análisis de los manuales en relación con las directrices del PCIC (Instituto Cervantes 2006). Así pues, se va a proceder a la revisión exhaustiva de siete manuales con características distintas y a la posterior valoración de los resultados extraídos.

La estructura de este artículo es la que sigue. En primer lugar, se recoge el marco teórico en el que se sintetizan las principales aportaciones en los estudios de la atenuación desde una perspectiva teórica y en su didáctica en E/LE. En segundo lugar, se presenta el estudio de campo, para el que se diseña una metodología de análisis. En tercer lugar, se recogen y analizan los resultados del análisis. Finalmente, se añadirá un apartado de anexos con el análisis detallado de los manuales y de los resultados.

\section{MARCO TEÓRICO}

\section{1. ¿Qué es la atenuación y cómo se reconoce?}

\subsubsection{Definición de atenuación como categoría pragmática}

En las últimas décadas, la investigación teórica en atenuación ha ido multiplicándose, y hoy en día se puede considerar como un fenómeno ciertamente definido y con una caracterización en gran manera estable por parte de la bibliografía (véase, por ejemplo, Caffi 1999, Briz 2007). Sin embargo, a pesar del impulso teórico que ha recibido, apenas ha sido desarrollado y aplicado al ámbito de la enseñanza de lenguas extranjeras, y más concretamente, en la enseñanza de ELE.

Entre las diversas definiciones del concepto, se ha acudido a una de las más citadas y que enmarca el fenómeno en el contexto de la pragmática, disciplina en la que mejor se ubica (Briz 2007): 
La atenuación [...] es una categoría pragmalingüística cuya función consiste en minimizar la fuerza ilocutiva de los actos de habla y el papel de los participantes de la enunciación con el fin de lograr la meta prevista, el acuerdo (o en minorar en su caso el desacuerdo), que es el fin último o primero, según se mire, de toda conversación. Quitar relieve, suavizar, mitigar, reparar, esconder la verdadera intención son valores que describen de forma más concreta esta operación lingüística estratégica vinculada a la actividad argumentativa y de negociación de dicho acuerdo, un acuerdo, que a veces es también social, de imagen, y en concreto a menudo de imagen cortés. [...] Metodológicamente, es necesario hacer la precisión anterior, porque la atenuación es una función estratégica que no siempre tiene que ver con las actividades de imagen cortés y ni siquiera a veces con la imagen. La atenuación, en todos los casos (con o sin cortesía), es un fenómeno semántico-pragmático (Briz 2007: 7-9)

Como se puede observar en esta definición, la atenuación se utiliza tanto para quitar importancia al contenido y a los matices negativos de un mensaje, como para encubrir la intención que realmente el hablante quiere expresar. Así pues, "la atenuación incide en el discurso de dos maneras: bien minimizando la fuerza ilocutiva de los actos de habla, bien reduciendo (y desfocalizando) el papel de los participantes en la enunciación (Briz 2007, Caffi 2007)".

Briz (2011) y Briz y Estellés (2010) explican que las funciones de un atenuante pueden ser incluidas en una de las tres siguientes: autoprotección, prevención y curación o reparación. Por su parte, en la "Ficha metodológica para el análisis pragmático de la atenuación en corpus discursivos del español. Es.Por.Atenuación" (Albelda, Briz, Cestero, Kotwica y Villalba 2014), explican que "se ha añadido la función 0 (autoprotección sin imagen), de manera provisional, pues no parece estar claro por parte de la bibliografía si puede emplearse la atenuación sin que medien cuestiones de imagen (Bravo 2005, 2008; Briz 2012)". Estas son las cuatro funciones de la atenuación, de acuerdo con la mencionada ficha metodológica:

Función 0. Velar por sí mismo evitando o reduciendo el compromiso del hablante con lo dicho, sin que medie directamente un interés de la imagen. Autoprotección sin imagen.

Función 1. Velar por sí mismo autoprotegiéndose por lo dicho o por lo hecho, con un interés de ganar o no perder imagen. Autoprotección con imagen. Salvaguarda del yo. Curarse en salud.

Función 2. Prevenir una posible amenaza a la imagen del otro o un posible obstáculo en la consecución de una meta. Prevención. Salvaguarda del yo y del tú.

Función 3. Reparar una amenaza a la imagen del otro o una intromisión en el territorio del otro. Curación. Salvaguarda del yo y del tú. (Albelda et alii. 2014: 8-12)

Así pues, la función 0 trata de reducir el compromiso del hablante sin tener en cuenta la protección de la imagen, mientras que las tres siguientes sí están relacionadas con las actividades de la imagen. De modo que, la primera función está relacionada con la intención del emisor de salvaguardar su imagen; la segunda, con la intención del emisor de velar por su imagen, al tiempo que previene al receptor de posibles amenazas; y, por último, la tercera está relacionada con reparar la imagen del otro.

Estas funciones se pueden reconocer gracias al contexto, ya que solo a través de este se pueden identificar las intenciones del hablante gracias a la aportación de los rasgos situacionales. También el contexto puede aportar otros datos, como, por ejemplo, si el mensaje es amenazante o no, según la relación existente entre ambos interlocutores. 
Respecto a las actividades de imagen, autores como Hernández Flores (2004), Bravo (2005, 2008), Briz (2007), Albelda y Briz (2010) o Holmlander (2011), coinciden y explican que en cuanto a las actividades de imagen y, en concreto, a la cortesía, la atenuación es uno de sus mecanismos de expresión lingüística. En otras palabras, de acuerdo con estos autores, la atenuación solo es uno de los modos de expresar cortesía, pero, en ningún caso, un sinónimo de cortesía. En este sentido, también el PCIC (Instituto Cervantes 2006) establece una clara distinción entre ambos conceptos (cortesía y atenuación). De acuerdo con estos autores, conviene distinguir entre atenuación (sin cortesía) y atenuación cortés.

\subsubsection{Criterios para reconocer la atenuación}

La atenuación es un fenómeno pragmático, no semántico, lo que implica que su valor está ligado a cálculos contextuales: una misma forma lingüística puede estar realizando la función de atenuante en un contexto determinado, mientras que en otro no. Para Albelda (2010) "el problema de la identificación de los enunciados atenuados estriba en que, a pesar de que sus formas y funciones se encuentran, con más o menos suerte, establecidas, la atenuación es una categoría pragmática y solo en su contexto real es posible identificarla (2010: 49)”.

En cuanto a los tipos de atenuantes, Briz $(2003,2007)$ clasifica los atenuantes en

a. Atenuación de lo dicho o del contenido proposicional, explicado por Briz (1995, 1998, 2003), en la que se atenúa también indirectamente el decir: es aquella por la que se minimiza la cantidad o se expresa un elemento de la proposición de forma vaga o imprecisa.

b. Atenuación directa del decir o de la fuerza ilocutiva de un acto de habla (Briz 1995, 1998, 2003). De acuerdo con la tipología clásica de actos de habla de Searle (1969), la atenuación puede afectar a los siguientes aspectos:

- actos asertivos: la atenuación en este tipo de actos se utiliza para eludir la responsabilidad y el compromiso del hablante;

- actos directivos: en beneficio del YO $\rightarrow$ expresan petición; en beneficio del TÚ $\rightarrow$ expresan consejo;

- actos comisivos (promesa e invitación): mediante la atenuación el hablante reduce el compromiso con sus promesas;

- actos expresivos: insultos, recriminaciones o quejas, cuando está en juego la imagen del interlocutor.

Briz (2006) y Briz y Albelda (2013) proponen una serie de factores que pueden ayudar al reconocimiento de los operadores de atenuación; mayoritariamente, constituyen rasgos situacionales. Los rasgos positivos $(+)$ son los que más invitan al uso de atenuantes:

1. Marco físico donde se realiza la entrevista o conversación:

(-) Familiar/cotidiano

(+) Transaccional

2. Relación de poder/ jerarquía entre los interlocutores:

(-) Igualdad funcional y/o social

(+) Desigualdad funcional y/o social 
3. Grado de proximidad, conocimiento común compartido entre los interlocutores:

(-) Relación vivencial de proximidad: amigos, parientes, colegas, conocidos

(+) Relación vivencial de no proximidad (desconocimiento)

4. Origen geográfico y cultural del hablante

(-) Cultura de acercamiento

(+) Cultura de distanciamiento.

Estos parámetros marcan la situación comunicativa; en el caso concreto del origen geográfico y cultural del hablante, Briz (2006) explica que, aunque la atenuación se expresa en todas las lenguas, la modalización depende de cada sociedad y cultura. Así pues, considera que la atenuación cortés puede tener un principio de explicación si se aborda a través de la distinción entre culturas de acercamiento y culturas de distanciamiento, y lo explica del siguiente modo:

+ atenuación cortés $\rightarrow-$ cultura de acercamiento

- atenuación cortés $\rightarrow+$ cultura de acercamiento

En culturas de menos acercamiento o de distanciamiento, la frecuente atenuación cortés es una estrategia de aproximación social al otro. Si con la atenuación, según señalábamos, estratégicamente intento acercarme al otro, es porque existe o construimos algún tipo de distancia entre el yo y el tú o entre las intenciones y metas de uno y de otro (Briz, 2006: 247-251).

\subsection{Cómo llevar al aula de ELE un fenómeno pragmático como la atenuación}

\subsubsection{La enseñanza/ aprendizaje formal/explícito en el aula del componente pragmático}

Hoy en día es impensable plantearse la didáctica de lenguas si no es desde la competencia comunicativa (Hymes 1976), esta es la capacidad de una persona para comportarse de manera eficaz y adecuada en una determinada comunidad de habla; ello implica respetar un conjunto de reglas que incluye tanto las de la gramática y los otros niveles de la descripción lingüística (léxico, fonética, semántica), como las reglas de uso de la lengua, relacionadas con el contexto sociohistórico y cultural en el que tiene lugar la comunicación (Hymes 1976).

Muchos autores describen la competencia comunicativa como un conjunto de competencias interrelacionadas, entre las que se encuentra la competencia pragmática ${ }^{1}$. Esta interrelación obedece a la necesidad de dominar la competencia pragmática para poder alcanzar la competencia comunicativa, ya que de la competencia pragmática depende que se haga un uso comunicativo adecuado, de acuerdo con las relaciones que se dan entre los signos lingüísticos y los interlocutores y el contexto comunicativo. Desde el punto de vista educativo, la competencia pragmática coincide con el desarrollo y adquisición de una adecuada competencia comunicativa, que se encuentra estrechamente relacionada con el enfoque comunicativo de la enseñanza de lenguas.

1 La competencia pragmática es uno de los componentes que algunos autores han descrito en la competencia comunicativa. Atañe a la capacidad de realizar un uso comunicativo de la lengua en el que se tengan presentes no solo las relaciones que se dan entre los signos lingüísticos y sus referentes, sino también las relaciones pragmáticas, es decir, aquellas que se dan entre el sistema de la lengua, por un lado, y los interlocutores y el contexto de comunicación por otro (Diccionario de términos clave de ELE, 2008). 
El modelo más representativo de competencia comunicativa es el de Bachman (1990), quien señala que la competencia comunicativa no se adquiere únicamente con el dominio de los conocimientos gramaticales, sino que necesita el desarrollo de competencias textuales y pragmáticas por parte del alumno.

No obstante, a la hora de la verdad, son múltiples las dificultades para aplicar la competencia pragmática en la enseñanza de lenguas, especialmente porque los paradigmas predominantes de didáctica de lenguas en los últimos siglos y hasta principios de los 80 del siglo XX eran de corte estructural. Asimismo, es complejo hacer consciente al estudiante de los recursos contextuales de los que depende el uso de la lengua, sobre todo cuando sus parámetros pragmático-culturales son diferentes a los del nativo. En ese sentido, Kasper (1997) y otros autores señalan que los estudiantes de L2, en el reconocimiento e identificación de los tipos de actos de habla, muestran una conciencia pragmática muy limitada. Es, por tanto, necesario hacer frente a la sensibilización para la enseñanza de la pragmática, tal y como ha apuntado Schmidt (1993).

Respecto a la enseñanza y aprendizaje del componente pragmático en el aula, Kasper (1997) apela a la posibilidad de que el conocimiento pragmático se desarrolle sin intervención pedagógica. Posteriormente, explica que esto se debe a que hay una parte del conocimiento pragmático que es universal (respetar los turnos de habla, las estructuras internas de los actos de habla y la importancia del contexto), y otra parte que puede ser trasferida a partir de la L1. No obstante, no siempre aprovechan la interferencia de la L1 y, por ello, en un segundo momento es necesaria la intervención pedagógica.

\subsubsection{La enseñanza/aprendizaje de la atenuación en el aula}

En tanto que el objeto de estudio de este trabajo es la atenuación en el ámbito de ELE, se recurre al MCER (Consejo de Europa, 2001) y al PCIC (Instituo Cervantes 2006) para ver qué mecanismos de atenuación corresponden a cada nivel de lengua. Una revisión de ambos documentos muestra que la atenuación no se incluye en el MCER (Consejo de Europa 2001) y que, en el caso del PCIC (Instituto Cervantes 2006) se trata dentro del apartado de cortesía, bajo el epígrafe "cortesía atenuadora".

Así pues, el PCIC (Instituto Cervantes 2006) considera que la atenuación es una táctica y estrategia pragmática que debería tratarse en el apartado de "Modalización”. Sin embargo, en este apartado hay una remisión al apartado de "Cortesía verbal atenuadora", puesto que, aunque no todos los mecanismos de atenuación sean estrategias de cortesía, se ha optado por desarrollar la atenuación en el apartado de la conducta interaccional para evitar repeticiones.

En el ámbito de la didáctica de lenguas no se encuentran muchas referencias que aborden la cuestión de cómo llevar al aula de ELE la atenuación. Kasper (1997) apunta que es muy importante considerar las oportunidades que nos ofrece la enseñanza tradicional de idiomas y explica que en las aulas de estudio tradicionales el profesor es quien realiza la mayor parte de la comunicación. Por tanto, es importante concienciarse de que el aprendizaje/enseñanza de una L2 debe basarse en el estudio de un medio necesario para la comunicación. En este sentido, Kasper extrae dos tipos de actividades útiles para desarrollar la competencia pragmática:

1. actividades centradas en la sensibilización pragmática de los estudiantes: en las que los alumnos adquieren información sociopragmática y pragmalingüística a través de la observación de rasgos pragmáticos particulares en fuentes orales y escritas. 
2. actividades que ofrecen oportunidades para la práctica comunicativa: interacciones en situaciones comunicativas que impulsen al estudiante a tomar tanto el papel de oyente como el de hablante.

Además, Kasper (1997) en su artículo cita a Peirce (1995) para explicar que las clases de lengua proporcionan un escenario ideal para explorar la relación entre la subjetividad de los alumnos y el uso L2, pues en un segundo idioma las aulas ofrecen a los estudiantes la oportunidad de reflexionar sobre los encuentros comunicativos y experimentar con diferentes opciones pragmáticas.

A partir de esta propuesta, Kasper se desliga del hecho de que, para un buen aprendizaje de la atenuación, esta debe ser explotada didácticamente y practicada, pues su estudio no se debe limitar a la observación de listas de uso o presencia de mecanismos en textos, sino que los estudiantes necesitan explotar los recursos y ponerlos en práctica para interiorizarlos.

\section{ESTUDIO DE CAMPO}

\subsection{Metodología de análisis}

El presente trabajo pretende llevar a cabo el análisis de un corpus de manuales de enseñanza de ELE de nivel B2. En ello, se analiza, por un lado, la presencia de recursos y funciones de atenuación y, por otro lado, la explotación didáctica que los autores del manual hacen de la atenuación. En concreto, se han seleccionado siete manuales sobre los que se ha realizado un análisis exhaustivo de este fenómeno. El análisis parte de un estudio previo de los parámetros establecidos por el MCER (Consejo de Europa 2001) y el PCIC (Instituto Cervantes 2006) para el nivel B2, que serán posteriormente comparados con el tratamiento e importancia que le dan los manuales a la atenuación.

\subsubsection{Selección del corpus de manuales}

A continuación, se detallarán los manuales que han sido objeto de análisis.

Para determinar el nivel de lengua al que aplicar este estudio, se ha tenido en cuenta tanto la habilidad comunicativa de los estudiantes como los contenidos del PCIC (Instituto Cervantes 2006). Se considera que un nivel B2 es el idóneo para este análisis, porque supone un desarrollo suficiente de la capacidad pragmática del hablante en español.

Los dos requisitos indispensables para su selección han sido su adaptación a las directrices del MCER (Consejo de Europa 2001) y que correspondan al nivel B2. Además de estos requisitos formales de partida, se han seleccionado manuales publicados en España en los últimos 10 años, que hayan sido concebidos con un enfoque comunicativo, por tareas u orientado a la acción. Entre todas las posibilidades que ofrecen las editoriales, se han seleccionado los más empleados actualmente en la docencia de ELE. Asimismo, también se ha incluido en la selección de los manuales, el criterio relativo a la finalidad de la enseñanza: español para fines generales (EFG)/ español para fines específicos (EFE). Así pues, dos de los siete manuales son de español para los negocios (EFE). Por último, se ha incluido un manual dedicado a la enseñanza de español a inmigrantes para observar también si existe alguna diferencia en el tratamiento de la atenuación, respecto a los otros manuales. 
Junto con los manuales objeto de análisis, se ha elaborado un corpus de control compuesto por manuales de corte más estructuralista, para poder observar si existe alguna diferencia en este aspecto en cuanto a la inclusión y explotación de la atenuación. En suma, como se aprecia, todas estas variables de selección dan como resultado un amplio abanico en la variedad de manuales, de manera que se obtiene una amplia representación del campo de materiales de ELE.

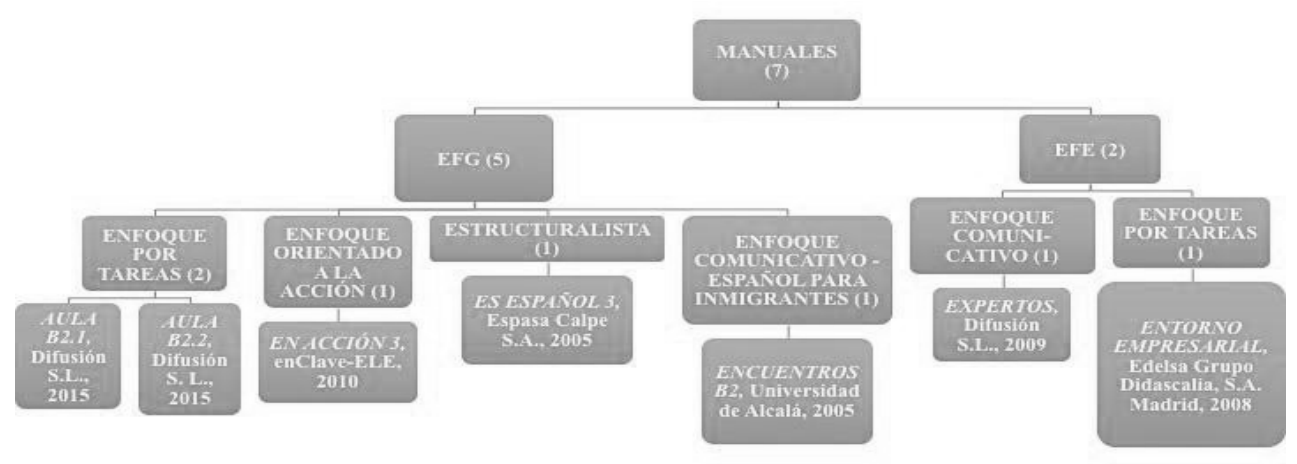

Imagen 1. Cuadro resumen presentación manuales.

\subsubsection{Criterios de análisis de la atenuación en el corpus de manuales}

El siguiente paso metodológico ha sido establecer los parámetros del análisis, es decir, qué partes y qué contenidos iban a ser analizados. Se ha dividido el análisis en tres dimensiones: índice, contenido de las unidades didácticas del manual y juicio crítico; que, a su vez, se dividen en partes menores. En el análisis de los manuales, se ha tenido en cuenta el índice y el contenido didáctico del manual para poder observar la coherencia o incoherencia de estos. El índice manifiesta los contenidos que se pretenden enseñar, pero en algunas ocasiones estos contenidos no corresponden con lo que después se plasma didácticamente.

En cuanto al análisis de las unidades didácticas, se han tenido en cuenta tres posibilidades, que el fenómeno /mecanismo de atenuación esté:

1. Explicado: si aparecen recursos de atenuación explicados mediante cuadros o anotaciones al margen.

2. Usado: si hay marcas de atenuación en los textos o actividades, pero que no se explican ni explotan didácticamente.

3. Explotado didácticamente: si se realizan actividades que trabajan en la forma o en el uso la atenuación.

La tercera dimensión de este análisis atañe al juicio crítico del analista, resultado del proceso de análisis, donde se estudia el aprovechamiento didáctico. En este punto, se han establecido dos posibles alternativas en el análisis: no se aprovecha la aparición de un fenómeno o sí se aprovecha (explicado y trabajado). 


\subsubsection{Nómina de mecanismos atenuantes analizados}

De acuerdo con el PCIC (Instituto Cervantes 2006), se ha establecido la siguiente tabla (Tabla 1), abajo, que servirá de guía en el análisis de los manuales de nivel B2:

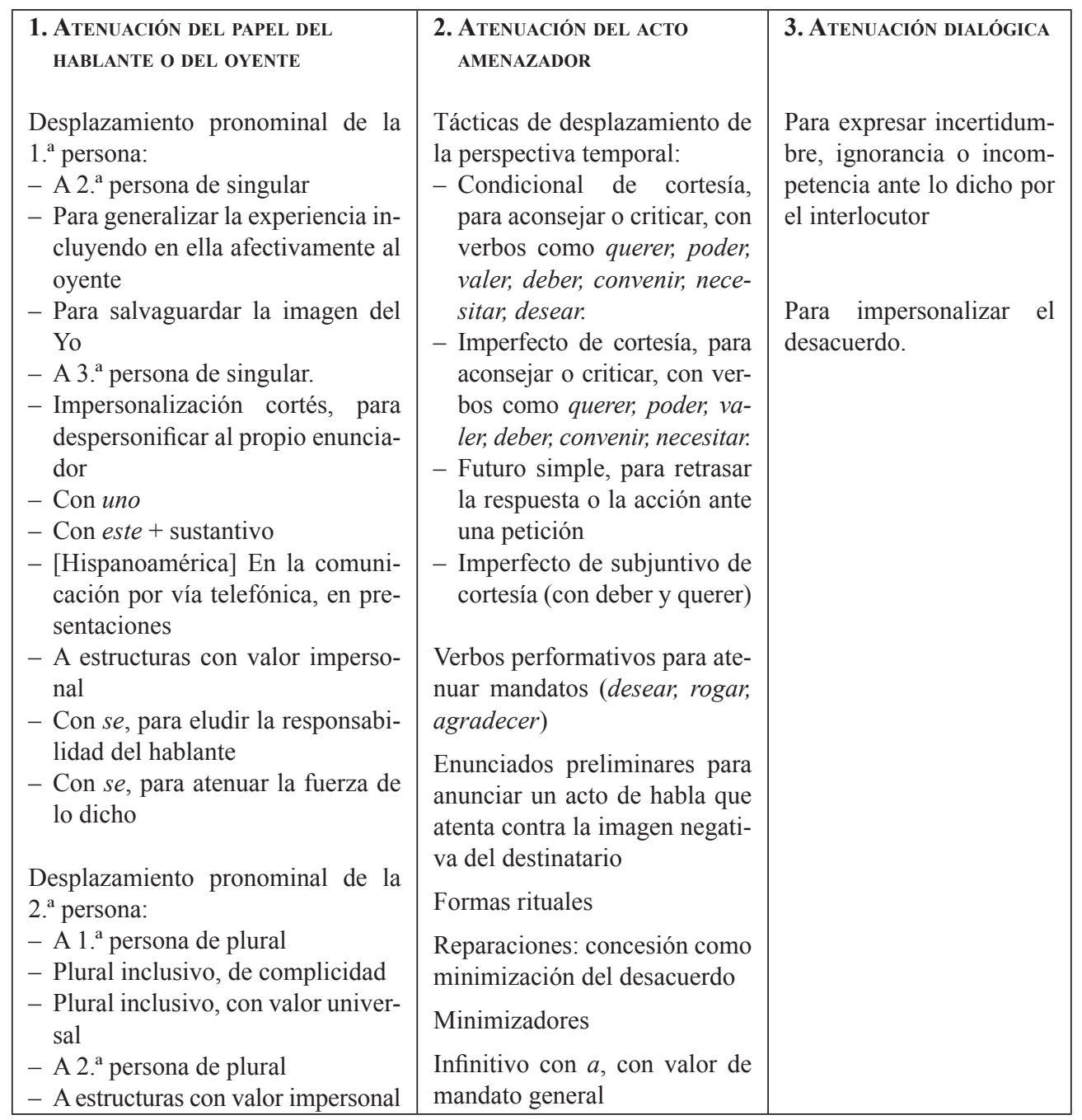

Tabla 1. Contenidos del PCIC (Instituto Cervantes 2006).

\subsubsection{Ficha final para el análisis de la atenuación de cada manual}

Una vez seleccionados los manuales y establecidos los parámetros y contenidos, se ha creado una ficha de análisis, dividida en tres bloques, de acuerdo con las partes delimitadas en el apartado anterior. 
La Tabla 2, abajo, muestra los resultados obtenidos en el índice. Siguiendo el orden visual (de izquierda a derecha y de arriba hacia abajo), los componentes de la tabla son los siguientes:

- Nombre manual: donde se indica el manual que se está analizando

- Índice: en este caso, el objeto de análisis

- Descripción: explicación del mecanismo de atenuación encontrado

- Página: número de la página donde se incluye un mecanismo de atenuación o uso de la atenuación (en un texto, actividad, etc.)

- Explícito (A): se selecciona esta casilla cuando el mecanismo de forma o uso de atenuación aparece explícito

- Implícito (B): se selecciona esta casilla cuando el mecanismo de forma o uso de atenuación se sobreentiende, pero no aparece explícito

Plantilla de análisis:

\begin{tabular}{|c|c|c|c|}
\hline Nombre manual & \multicolumn{2}{|c|}{ Índice } & \multirow{2}{*}{ Descripción } \\
\hline Página & Explícito (A) & Implícito (B) & \\
\hline
\end{tabular}

Tabla 2. Plantilla índice.

Los componentes de la Tabla 3 (abajo) son estos:

- Nombre manual: donde se indica el manual que se está analizando

- Libro: en este caso, el objeto de análisis

- Descripción: explicaciones del mecanismo de atenuación encontrado

- Página: número de la página donde se incluye un mecanismo de atenuación o uso de la atenuación (en un texto, actividad, etc.)

- Explicado (C): se selecciona esta casilla si aparecen recursos de atenuación explicados mediante cuadros o anotaciones al margen

- Usado (D): se selecciona esta casilla si hay marcas de atenuación en los textos, pero no se trabajan

- Explotado didácticamente (E): se selecciona esta casilla si se realizan actividades que trabajan didácticamente la atenuación

Plantilla de análisis:

\begin{tabular}{|c|c|c|c|c|}
\hline Nombre manual & \multicolumn{3}{|c|}{ Contenido didáctico del manual } & Descripción \\
\hline \multirow{2}{*}{ Página } & $\begin{array}{c}\text { Explicado } \\
\text { (C) }\end{array}$ & $\begin{array}{c}\text { Usado } \\
\text { (D) }\end{array}$ & $\begin{array}{c}\text { Explotado } \\
\text { didácticamente } \\
\text { (E) }\end{array}$ & \\
& & & & \\
\hline
\end{tabular}

Tabla 3. Plantilla contenido didáctico del manual.

Los componentes de la Tabla 4 (abajo) son los que siguen:

- Nombre manual: título

- Juicio crítico: en este caso, el objeto de análisis, que evalúa el aprovechamiento didáctico 
- Unidad didáctica: el número de la unidad didáctica donde se incluyen los mecanismos de atenuación o uso de la atenuación (texto, actividad, etc.)

- Juicio crítico negativo: se considera un juicio negativo cuando en un fragmento del manual se registra el uso de mecanismos de atenuación, pero no se explican o se explotan didácticamente.

- Juicio crítico positivo: se considera un juicio positivo cuando en el manual aparece algún cuadro explicativo con los fenómenos de atenuación o actividades que explotan didácticamente estos mecanismos.

Plantilla de análisis:

\begin{tabular}{|c|c|c|}
\hline Nombre manual & \multicolumn{2}{|c|}{ Juicio crítico } \\
\hline Unidad & $\begin{array}{c}\text { Juicio crítico } \\
\text { positivo }\end{array}$ & Juicio crítico negativo \\
\hline
\end{tabular}

Tabla 4. Plantilla juicio crítico.

\subsection{Análisis y resultados}

\subsubsection{Análisis y resultados en los manuales B2}

Los resultados del análisis van a ser presentados en cinco apartados:

A. Resultados por manuales

De acuerdo con la metodología establecida (§ 3.1.), se recogen los resultados del siguiente modo:

\begin{tabular}{|c|c|c|c|}
\hline $\begin{array}{c}\text { PÁGINAS } \\
\text { ANALIZADAS }\end{array}$ & EXPLICADO (C) & USADO (D) & $\begin{array}{c}\text { EXPLOTADO } \\
\text { DIDÁCTICAMENTE } \\
(\mathrm{E})\end{array}$ \\
\hline
\end{tabular}

Tabla 5. Ficha análisis relación de ítems por manual.

Manual 1: Aula 2.1

\begin{tabular}{|c|c|c|c|}
\hline $\begin{array}{c}\text { PÁGINAS } \\
\text { ANALIZADAS }\end{array}$ & EXPLICADO $(\mathrm{C})$ & USADO (D) & $\begin{array}{c}\text { EXPLOTADO } \\
\text { DIDÁCTICAMENTE } \\
(\mathrm{E})\end{array}$ \\
\hline 128 & 13 & 7 & 26 \\
\hline
\end{tabular}

Tabla 6. Resultados Aula B2.1. 
Se observa que la valoración general de Aula B2.1 es positiva. En 128 páginas aparecen 13 explicaciones relacionadas con la atenuación y 26 actividades en las que se explotan estos mecanismos, frente a los 7 usos encontrados en textos y actividades, que no han sido explotados didácticamente.

Manual 2: Aula B2.2

\begin{tabular}{|c|c|c|c|}
\hline $\begin{array}{c}\text { PÁGINAS } \\
\text { ANALIZADAS }\end{array}$ & EXPLICADO $(\mathrm{C})$ & USADO (D) & $\begin{array}{c}\text { EXPLOTADO } \\
\text { DIDÁCTICAMENTE } \\
(\mathrm{E})\end{array}$ \\
\hline 128 & 5 & 8 & 13 \\
\hline
\end{tabular}

Tabla 7. Resultados Aula B2.2.

En este manual también se han analizado un total de 128 páginas y los resultados que se han obtenido son: la presencia de 5 ítems explicados y 13 explotados didácticamente, frente a los 8 usados. Por tanto, la valoración del manual es positiva.

\section{Manual 3: Encuentros B2}

\begin{tabular}{|c|c|c|c|}
\hline $\begin{array}{c}\text { PÁGINAS } \\
\text { ANALIZADAS }\end{array}$ & EXPLICADO (C) & USADO (D) & $\begin{array}{c}\text { EXPLOTADO } \\
\text { DIDÁCTICAMENTE } \\
(\mathrm{E})\end{array}$ \\
\hline 85 & 5 & 9 & 23 \\
\hline
\end{tabular}

Tabla 8. Resultados Encuentros B2.

Encuentros $B 2$ pese a ser un manual más reducido, en cuanto al número de páginas, que los dos anteriores (85 páginas), presenta un total de 5 explicaciones y 23 actividades de explotación didáctica, frente a los 9 usos de mecanismos atenuantes que aparecen en textos y actividades sin ser trabajados. Se extrae, por tanto, una valoración final muy positiva.

Manual 4: Es Español 3

\begin{tabular}{|c|c|c|c|}
\hline $\begin{array}{c}\text { PÁGINAS } \\
\text { ANALIZADAS }\end{array}$ & EXPLICADO $(\mathrm{C})$ & USADO (D) & $\begin{array}{c}\text { EXPLOTADO } \\
\text { DIDÁCTICAMENTE } \\
(\mathrm{E})\end{array}$ \\
\hline 238 & 11 & 25 & 3 \\
\hline
\end{tabular}

Tabla 9. Resultados Es Español 3.

El manual Es Español 3 cuenta con 238 páginas, en las cuales aparecen 11 mecanismos explicados y 3 explotados didácticamente, frente a los 25 que han sido usados en textos y actividades sin ser explicados. Por lo que la valoración de este manual es negativa, respecto a las anteriores. 
MANUAL 5: EXPERTOS

\begin{tabular}{|c|c|c|c|}
\hline $\begin{array}{c}\text { PÁGINAS } \\
\text { ANALIZADAS }\end{array}$ & EXPLICADO (C) & USADO (D) & $\begin{array}{c}\text { EXPLOTADO } \\
\text { DIDÁCTICAMENTE } \\
(\mathrm{E})\end{array}$ \\
\hline 160 & 4 & 6 & 6 \\
\hline
\end{tabular}

Tabla 10. Resultados Expertos.

Dentro de las 160 páginas se han registrado 4 explicaciones y 6 explotaciones didácticas, frente a la presencia de 6 usos en textos. Es por esto que, se considera que la valoración de este manual es positiva.

Manual 6: Entorno empresarial

\begin{tabular}{|c|c|c|c|}
\hline $\begin{array}{c}\text { PÁGINAS } \\
\text { ANALIZADAS }\end{array}$ & EXPLICADO (C) & USADO (D) & $\begin{array}{c}\text { EXPLOTADO } \\
\text { DIDÁCTICAMENTE } \\
(\mathrm{E})\end{array}$ \\
\hline 121 & 0 & 16 & 0 \\
\hline
\end{tabular}

Tabla 11. Resultados Entorno empresarial.

De las 121 páginas analizadas, se han registrado únicamente 16 usos de mecanismos atenuantes, los cuales no aparecen explicados ni explotados didácticamente. Por lo que la valoración de este manual es negativa respecto a los anteriores. Es, por tanto, el manual que menos desarrolla la atenuación.

Manual 7: En acción 3

\begin{tabular}{|c|c|c|c|}
\hline $\begin{array}{c}\text { PÁGINAS } \\
\text { ANALIZADAS }\end{array}$ & EXPLICADO (C) & USADO (D) & $\begin{array}{c}\text { EXPLOTADO } \\
\text { DIDÁCTICAMENTE } \\
(\mathrm{E})\end{array}$ \\
\hline 177 & 3 & 7 & 9 \\
\hline
\end{tabular}

Tabla 12. Resultados En acción 3.

En este manual se han analizado 177 páginas. En ellas se ha observado la presencia de 3 mecanismos explicados y 9 explotados didácticamente, frente a los 7 que han sido usados sin explicación ni explotación. La valoración que se extrae de este manual es positiva.

B. Manual enfoque por tareas vi. Manual estructuralista

Como ya se ha explicado anteriormente ( $\$ 3.1 .1$.), se han seleccionado manuales concebidos con un enfoque comunicativo, orientado a la acción o por tareas. No obstante, para 
poder observar si existe alguna diferencia en este aspecto en cuanto a la metodología didáctica desde la que se conciben los diversos manuales, se ha elaborado un corpus de control compuesto por manuales de corte más estructuralista.

De la comparación entre un manual de enfoque por tareas (Aula B2.1) y uno de corte más estructuralista (Es Español 3) se extrae que:

- en el primero de ellos, pese a tener menos páginas, se explican dos mecanismos más que en el segundo (Aula B2.1 $\rightarrow \mathrm{C}=13$ vs. Es Español $3 \rightarrow \mathrm{C}=11$ ).

- en Aula B2.1 se registran 7 usos y 26 explotaciones didácticas $(\mathrm{D}=7, \mathrm{E}=26)$

- en Es Español 3 se anotan 25 usos y 3 explotaciones didácticas (D = 25, E = 3).

Estos resultados muestran claramente que los manuales de corte estructuralista, aunque sí se adaptan al PCIC (Instituto Cervantes, 2006) en cuanto a los contenidos, no explotan adecuadamente los mecanismos de atenuación.

\section{Español para fines Generales (EFG) vs. Español PARA fines esPecíficos (EFE)}

Tal y como se ha señalado en el apartado $§ 3.1 .1$, en la selección del corpus de manuales también se ha incluido el criterio relativo a la finalidad de la enseñanza: español para fines generales (EFG)/ español para fines específicos (EFE).

Así pues, se compararán en este apartado los resultados de un manual de español para fines generales (En acción 3) y otro para fines específicos (Expertos).

Entre ellos presentan casi una correspondencia exacta respecto al número de páginas analizadas y los resultados referentes a la explicación (C), uso (D) y explotación didáctica de los mecanismos de atenuación (E), pues la única diferencia notable reside en la presencia de 3 mecanismos más explotados didácticamente en Expertos, datos que, en un análisis de tales dimensiones, no resultan tan simbólicos como para establecer una diferencia entre ambos manuales. Por lo que se concluye que, la finalidad de la enseñanza no es un rasgo distintivo, como sí lo es la diferencia entre un manual con enfoque por tareas y uno de corte más estructuralista.

\section{Manual ENFoque por tareas vs. Español para inMigrantes}

Se comparan en este apartado los resultados de un manual de enfoque por tareas (Aula B2.2) con el de español para inmigrantes (Encuentros B2). Para ello, se recogen los resultados expuestos en el apartado anterior, concretamente las Tablas 7 y 8, correspondientes a los manuales Aula B2.2 y Encuentros B2, respectivamente.

Se obtiene en esta comparativa una valoración muy positiva respecto al manual de enseñanza de español para inmigrantes (Encuentros B2), pues pese a contar con 43 páginas menos que Aula B2.2, registra los mismos resultados en cuanto a C (explicado) y D (usado), pero registra 10 explotaciones didácticas (E) más de mecanismos de atenuación (Aula B2.2 $\rightarrow \mathrm{E}=13$ vs. Encuentros $B 2 \rightarrow \mathrm{E}=23$ ).

\section{E. RESUMEN CON TABLAS Y GRÁFICAS}

Véase a continuación la Tabla 13, abajo, con los resultados absolutos del total de los manuales para, después, poder realizar gráficas representativas de ellos (véanse los anexos). 
Además del análisis por manuales, detallado a priori, en la Tabla 13 se ejemplifican la coherencia entre el índice y el manual y los resultados de todas las dimensiones del análisis.

\begin{tabular}{|c|c|c|c|c|c|c|c|c|}
\hline MANUAL & $\begin{array}{l}\text { No PÁGINAS } \\
\text { ANALIZADAS }\end{array}$ & $\begin{array}{l}\text { EXPLÍ́CITO } \\
\text { (A) }\end{array}$ & $\begin{array}{l}\text { IMPLÍCITO } \\
\text { (B) }\end{array}$ & $\begin{array}{l}\text { EXPLICADO } \\
\text { (C) }\end{array}$ & $\begin{array}{l}\text { USADO } \\
\text { (D) }\end{array}$ & $\begin{array}{c}\text { EXPLOTADO } \\
\text { DIDÁCTICAMENTE } \\
\text { (E) }\end{array}$ & $\begin{array}{c}\text { JUICIO } \\
\text { CRÍTICO } \\
\text { NEGATIVO } \\
\text { (F) }\end{array}$ & $\begin{array}{c}\text { JUICIO } \\
\text { CRÍTICO } \\
\text { POSITIVO } \\
\text { (G) }\end{array}$ \\
\hline$A U L A B 2.1$ & 128 & 7 & 3 & 13 & 7 & 26 & 7 & 39 \\
\hline$A U L A B 2.2$ & 128 & 3 & 6 & 5 & 8 & 13 & 8 & 18 \\
\hline ENCUENTROS B2 & 85 & 6 & 12 & 5 & 9 & 23 & 9 & 28 \\
\hline ES ESPAÑOL 3 & 238 & 7 & 7 & 11 & 25 & 3 & 25 & 14 \\
\hline EXPERTOS & 160 & 5 & 3 & 4 & 6 & 6 & 6 & 10 \\
\hline $\begin{array}{c}\text { ENTORNO } \\
\text { EMPRESARIAL }\end{array}$ & 121 & 0 & 0 & 0 & 16 & 0 & 16 & 0 \\
\hline EN ACCIÓN 3 & 117 & 14 & 12 & 3 & 7 & 19 & 7 & 22 \\
\hline RESULTADOS: & 977 & 42 & 43 & 41 & 78 & 90 & 78 & 131 \\
\hline
\end{tabular}

Tabla 13. Resultados por manuales.

Respecto a la coherencia entre el índice y el manual, en la Tabla 13, si se comparan los resultados de los parámetros $\mathrm{A}$ (explícito) y $\mathrm{B}$ (implícito) con los de $\mathrm{E}$ (explotado didácticamente), se observa una coherencia, se podría decir, absoluta entre el índice y el manual:

$$
\begin{gathered}
\mathrm{A}+\mathrm{B}=\mathrm{X} \rightarrow 42+43=85 \\
\mathrm{AB}=85 \text { vs. } \mathrm{E}=90
\end{gathered}
$$

En cuanto a las dimensiones del análisis, se puede apreciar una valoración positiva respecto al tratamiento de los mecanismos de atenuación, pues, aunque muchos de ellos aparezcan usados $(\mathrm{D}=78)$, sin ser explicados ni explotados, se ha registrado un total de 90 recursos explotados didácticamente $(E=90)$, sumados a los 41 que han sido explicados $(C=41)$ :

$$
\begin{gathered}
\mathrm{C}+\mathrm{E}=\mathrm{X} \rightarrow 41+90=131 \\
\mathrm{CE}=131 \text { vs. } \mathrm{D}=78
\end{gathered}
$$

\subsubsection{Comparación entre manuales y PCIC}

Llegado a este punto, se realiza la comparación de los resultados de los manuales con el PCIC (Instituto Cervantes, 2006). No se va a realizar una comparación exhaustiva de los contenidos, sino que se va a partir de que todos los manuales cumplen y siguen el PCIC (Instituto Cervantes, 2006). Por tanto, se comentarán únicamente los contenidos y casos especiales que muestren una clara incoherencia.

De acuerdo con los contenidos expuestos en $\S 3.1 .3$ (Tabla 1), en el nivel B2 se tratan aproximadamente once mecanismos de atenuación. Así pues, se va a considerar como valoración positiva que los manuales exploten didácticamente al menos seis de ellos. A cada uno de estos mecanismos se le ha asignado una letra y, posteriormente, se ha realizado una 
tabla como la de abajo (Tabla 14). Los resultados se expresan mediante los signos "X" $\mathrm{y}$ “-”, donde "-" representa la ausencia del mecanismo explotado didácticamente en el manual, y "X", su presencia. Los mecanismos de atenuación son los siguientes:
A. Desplazamiento pronominal de la $1 .^{\text {a }}$ persona
B. Desplazamiento pronominal de la 2. ${ }^{\text {a }}$ persona
C. Tácticas de desplazamiento de la perspectiva temporal
D. Verbos performativos para atenuar mandatos (desear, rogar, agradecer)
E. Enunciados preliminares para anunciar un acto de habla que atenta contra la imagen negativa del destinatario
F. Formas rituales
G. Reparaciones: concesión como minimización del desacuerdo
H. Minimizadores
I. Infinitivo con $a$, con valor de mandato general
J. Para expresar incertidumbre, ignorancia o incompetencia ante lo dicho por el inter- locutor
K. Para impersonalizar el desacuerdo.

\begin{tabular}{|l|c|c|c|c|c|c|c|c|c|c|c|c|}
\hline $\begin{array}{l}\text { CONTENIDO } \\
\text { MANUAL }\end{array}$ & $\mathrm{A}$ & $\mathrm{B}$ & $\mathrm{C}$ & $\mathrm{D}$ & $\mathrm{E}$ & $\mathrm{F}$ & $\mathrm{G}$ & $\mathrm{H}$ & $\mathrm{I}$ & $\mathrm{J}$ & $\mathrm{K}$ & TOTAL \\
\hline AULA B2.1 & $\mathrm{X}$ & $\mathrm{X}$ & $\mathrm{X}$ & $\mathrm{X}$ & - & - & $\mathrm{X}$ & $\mathrm{X}$ & - & $\mathrm{X}$ & $\mathrm{X}$ & 8 \\
\hline AULA B2.2 & - & $\mathrm{X}$ & $\mathrm{X}$ & - & - & - & $\mathrm{X}$ & $\mathrm{X}$ & - & $\mathrm{X}$ & $\mathrm{X}$ & 6 \\
\hline ENCUENTROS B2 & $\mathrm{X}$ & $\mathrm{X}$ & $\mathrm{X}$ & $\mathrm{X}$ & - & $\mathrm{X}$ & - & $\mathrm{X}$ & - & $\mathrm{X}$ & $\mathrm{X}$ & 8 \\
\hline ES ESPAN OL 3 & $\mathrm{X}$ & $\mathrm{X}$ & $\mathrm{X}$ & $\mathrm{X}$ & - & - & $\mathrm{X}$ & $\mathrm{X}$ & - & - & $\mathrm{X}$ & 7 \\
\hline EXPERTOS & - & $\mathrm{X}$ & $\mathrm{X}$ & - & - & - & - & - & - & $\mathrm{X}$ & $\mathrm{X}$ & 4 \\
\hline ENTORNO EMPRESARIAL & - & - & $\mathrm{X}$ & - & - & - & - & - & - & - & - & 1 \\
\hline EN ACCIÓN 3 & $\mathrm{X}$ & $\mathrm{X}$ & $\mathrm{X}$ & - & - & - & $\mathrm{X}$ & $\mathrm{X}$ & - & $\mathrm{X}$ & $\mathrm{X}$ & 7 \\
\hline
\end{tabular}

Tabla 14. Presencia de contenidos atenuantes en los manuales según el PCIC (Instituto Cervantes, 2006).

De acuerdo con la Tabla 14, se puede ver que:

- cinco de los siete manuales (5/7) tienen presentes entre seis y ocho mecanismos $\rightarrow$ $5 / 7(6-8)$

- uno de los manuales (1/7) presenta entre cuatro y seis mecanismos $\rightarrow 1 / 7$ (4-6)

- uno de los manuales tiene presentes menos de cuatro mecanismos $\rightarrow 1 / 7$ (-4)

De estos resultados se extrae que los manuales Aula B2.1, Aula B2.2, Encuentros B2, Es Español 3 y En acción 3 se adaptan mejor al PCIC (Instituto Cervantes 2006) que los manuales Expertos y Entorno empresarial. Se observa también que, a diferencia de la comparativa realizada anteriormente en el apartado C (§3.2.3., Tablas 10 y 12), en la que únicamente se tenía en cuenta la explotación didáctica de mecanismos, sí existe una diferencia clara, en cuanto a los contenidos, entre los manuales de EFG y EFE, pues los de EFE se centran únicamente en los contenidos relacionados con dar consejos y ofrecer ayuda. 


\subsubsection{Valoración de los resultados del análisis}

De todos los resultados ${ }^{2}$ y comparativas establecidas entre los manuales en $\S 3.2 .3$., se extrae, de acuerdo con los diferentes apartados de ese mismo punto, que:

- de los resultados del análisis por manuales se obtiene una valoración positiva de cinco manuales, frente a una valoración negativa de los dos restantes;

- de la comparativa entre un manual de enfoque por tareas y uno de corte más estructuralista, se extrae una valoración negativa del manual estructuralista, pues, aunque en contenidos se adapta al PCIC (Instituto Cervantes 2006), no los explota didácticamente;

- de la comparativa entre manuales de EFG y manuales de EFE no se obtiene ningún rasgo distintivo que ataña a la finalidad de la enseñanza;

- de la comparativa entre un manual de enfoque por tareas y uno de español para inmigrantes se extrae una valoración muy positiva respecto al manual de español para inmigrantes;

- de la comparativa con el PCIC (Instituto Cervantes 2006) se extrae que cinco de los siete manuales registran la presencia de al menos seis mecanismos de atenuación, así como una diferencia entre los manuales de EFG y EFE.

\section{CONCLUSIONES}

El trabajo que se ha llevado a cabo ha permitido estudiar y analizar el tratamiento de un fenómeno tan importante como es la atenuación en la enseñanza de español para extranjeros, a través de los manuales de nivel B2 y la comparación de estos con las directrices del PCIC (Instituto Cervantes 2006).

En primer lugar, se ha estudiado la atenuación como categoría pragmática, así como los criterios que permiten reconocerla. En segundo lugar, se ha intentado definir la manera más adecuada de llevar un fenómeno pragmático al aula de ELE y la importancia de despertar la conciencia pragmática. En tercer lugar, se ha procedido a elaborar un estudio de campo, a partir del cual se ha seleccionado un corpus de manuales, se han establecido unos criterios de análisis, se ha seleccionado una nómina de mecanismos atenuantes, de acuerdo con el PCIC (Instituto Cervantes, 2006), y se ha elaborado una ficha de análisis. Finalmente, se han analizado y comparado los resultados.

Una vez llevado a cabo todo el proceso de análisis y extracción de resultados, tal y como se muestra en $\S 3.3 .3$, se concluye que los manuales analizados obtienen una valoración positiva. No obstante, es necesario apuntar que no existe el manual perfecto, puesto que en el análisis se ha observado que hay manuales que se adaptan muy bien a las directrices del PCIC (Instituto Cervantes 2006), como son: Aula B2.1, Aula B2.2, Encuentros B2, Es Español 3 y En acción 3, pero no obtienen una valoración positiva en cuanto al tratamiento o explotación didáctica del fenómeno, como es el caso de Es Español 3, por ejemplo. También se observa lo contrario, que otros manuales como Expertos, aunque explotan muy bien didácticamente la atenuación, únicamente desarrollan 4 de los 11 contenidos del PCIC (Instituto Cervantes 2006).

2 Se recuerda que la valoración se considera positiva si los contenidos están explicados o explotados didácticamente, y se considera negativa cuando los contenidos se usan sin explicar o explotar. 
Por tanto, de este análisis se extrae que ninguno de los manuales analizados cubre todas las expectativas previstas de inclusión de mecanismos atenuantes y adecuada explotación didáctica de ellos. Asimismo, ningún manual cubre totalmente las indicaciones del PCIC (Instituto Cervantes 2006) en cuanto a la cortesía atenuadora concierne. Así pues, este estudio muestra la necesidad de mejorar la didáctica de la atenuación, y por extensión de los diversos fenómenos pragmáticos, por parte de los autores de materiales didácticos y de profesores de ELE. Los datos ofrecidos por la presente investigación deberían servir para iniciar una línea entre los estudiosos en ELE en el campo de la atenuación y proponer modos y maneras de enseñar con más atención sus formas y sus funciones, lo que, a su vez, llevará a mejorar la conciencia pragmática en los estudiantes de ELE.

\section{Referencias bibliográficas}

Albelda, M. (2008). “Atenuantes en Chile y en España: distancia o acercamiento". En Briz, A., Hidalgo, A., Albelda, M., Contreras, J. y Hernández Flores, N. (eds.), Cortesía y conversación: de lo escrito a lo oral, pp. 98-113: http://www.uv.es/aleza/Cap.\%205.\%20EA\%20Prag.pdf (06-12-2015).

Albelda, M. (2010). “¿Cómo se reconoce la atenuación? Una aproximación metodológica basada en el español peninsular hablado". En Orletti, F. y L. Mariottini (eds.). (Des)cortesía en español. Roma: Università Roma Tre, pp. 41-70.

Albelda, M. y A. M. Cestero (2011). "De nuevo, sobre los procedimientos de atenuación”, Español actual, 96, pp. 121-155.

Albelda, M. y A. M. Cestero (2012). "La atenuación lingüística como fenómeno variable". En Cestero, A. M., Molina, I. y F. Paredes (eds.), La lengua, lugar de encuentro. Actas XVI Congreso Internacional de la Alfal (Alcalá de Henares, 6-9 de junio de 2011). Alcalá de Henares: Servicio de Publicaciones de la Universidad de Alcalá.

Albelda, M. y M. J. Barros (2013). La cortesía en la comunicación. Madrid: Arco/Libros.

Albelda, M., Briz, A., Cestero, A. M., Kotwica, D., y C. Villalba (2014). "Ficha metodológica para el análisis pragmático de la atenuación en corpus discursivos del español. (ES.POR. ATENUACIÓN)", Oralia, 17, pp. 7-62.

Alcón, E. y M.P. Safont (2008). "Pragmatic awareness in second language acquisition”. En Nancy H. Hornberger (ed.). Encyclopedia of Language and Education. Berlín: Editorial Springer Link, pp. 1948-1959.

Bachman, L. (1990). "Habilidad lingüística comunicativa". En Llobera (coord.), Competencia comunicativa. Documentos básicos en la enseñanza de lenguas extranjeras. Madrid: Edelsa, pp. 105-129.

Bartol, E. (2009). "Es que no se me ocurría nada mejor: La función pragmático-comunicativa del rechazo cortés a las invitaciones y propuestas". Universidad de Salamanca. Memoria final de máster: https://www.mecd.gob.es/educacion/mc/redele/biblioteca-virtual/numerosanteriores/2012/ memoria-master/estela-bartol.html (07/12/2015).

Bravo, D. (2005). "Categorías, tipologías y aplicaciones. Hacia una redefinición de la 'cortesía comunicativa"'. En D. Bravo (ed.). Estudios de la (des)cortesía en español. Categorías conceptuales y aplicaciones a corpora orales y escritos. Buenos Aires: Dunken, pp. 21-52.

Bravo, D. (2008). "Situación de habla, recursos comunicativos y factores lingüísticos en la interpretación de objetivos de cortesía". En Briz, A., Hidalgo, A., Albelda, M., Contreras, J. y Hernández Flores, N. (eds.), Cortesía y conversación: de lo escrito a lo oral. Valencia: Universitat de València, pp. 12-24.

Briz, A. (1998). El español coloquial en la conversación. Esbozo de pragmalingiiística. Barcelona: Ariel.

Briz, A. (2006). "Atenuación y cortesía verbal en la conversación coloquial: su tratamiento en la clase de ELE". Actas del programa de formación para profesorado de ELE. Munich: Instituto Cervantes, pp. 227-255. 
Briz, A. (2007). "Para un análisis semántico, pragmático y sociopragmático de la cortesía atenuadora en España y América”, LEA, XIX, pp. 1-38.

Briz, A. (2011). "La atenuación como categoría pragmática". En StrosetzkiC. (ed.), Actas del VII Congreso Internacional de la Asociación Asiática de Hispanistas. Pekín: Foreign Language Teaching and Research Press.

Briz, A. (2012). “La (no) atenuación y la (des)cortesía, lo lingüístico y lo social: ¿son pareja?”. En Escamilla Morales, J. y Henry Vega, G. (eds.). Miradas multidisciplinares a los fenómenos de cortesía y descortesía en el mundo hispánico. Barranquilla: Universidad del Atlántico-Programa EDICE, pp. 33-75.

Briz, A., y M. Estellés (2010). "On the relationship between Attenuation. Discourse Particles and Position", Studies in Pragmatics, 9, pp. 289-304.

Caffi, C. (1999). “On mitigation”, Journal of Pragmatics, 31, pp. 881-909.

Canale, M. (1995 [1983]). "De la competencia comunicativa a la pedagogía comunicativa de la lengua”. En Llobera (coord.). Competencia comunicativa. Documentos básicos en la enseñanza de lenguas extranjeras. Madrid: Edelsa, pp. 63-82.

Consejo de Europa (2001). Marco común europeo de referencia de las lenguas. Instituto Cervantes, para la traducción en español: http://cvc.cervantes.es/obref/marco (01/12/2015).

Felix-Brasdefer, J. C. (2004). "La mitigación en el discurso oral de mexicanos y aprendices de español como lengua extranjera". En Briz, A. y D. Bravo (coords.). Pragmática sociocultural: estudios sobre el discurso de cortesía en español. Barcelona: Ariel, pp. 285-299.

Hernández Flores, N. (2004). "La cortesía como búsqueda del equilibrio de la imagen social". En Bravo, D. y A. Briz (eds.). Pragmática sociocultural: estudios sobre el discurso de cortesía en español. Barcelona: Ariel, pp. 95-108.

Holmlander, D. (2008). “Atenuación con y sin cortesía. Un estudio de conversaciones interculturales entre españoles y suecos". Lund: Lund University Publications: https://www.academia. edu/2107936/Atenuaci\%C3\%B3n_con_y_sin_cortes\%C3\%ADa._Un_estudio_de_conversaciones_interculturales_entre_espa\%C3\%B1oles_y_suecos $(05 / 02 / 2016)$.

Holmlander, D. (2011). Estrategias de atenuación es español L1 y L2. Estudio contrastivo en hablantes españoles y suecos. Suecia: Lunds Universitet.

Hymes, D. (1976). "On communicative competence”. En J.B. Pride y J. Holmes (eds.). Sociolinguistics, Harmondworth: Penguin, pp. 269-293..

Instituto Cervantes (2006). Plan Curricular del Instituto Cervantes. Niveles de referencia para el español. Madrid: Instituto Cervantes. Editorial Biblioteca Nueva.

Instituto Cervantes (2008). Diccionario de términos clave de ELE. Madrid: Instituto Cervantes, SGEL.

Kasper, G. (1997). "Can pragmatic competence be taught?”. Honolulu: University of Hawai'i, Second Language Teaching \& Curriculum Center: http://www.nflrc.hawaii.edu/NetWorks/NW06/ $(01 / 03 / 2016)$.

Landone, E. (2009). "Reflexiones sobre la cortesía verbal en la enseñanza / aprendizaje del ELE", Marcoele, 8: http://marcoele.com/descargas/8/landone_cortesia.pdf (06/12/2015).

Medina, I. (2012). "Los elementos atenuadores para expresar desacuerdo en el discurso oral de estudiantes E/LE universitarios de nivel B1 en contexto de inmersión. Estudio cualitativo", Revista Nebrija de Lingüistica Aplicada a la Enseñanza de Lenguas, 11(6), pp. 104-143: http://www.nebrija.com/revista-linguistica/los-elementos-atenuadores-para-expresar-desacuerdo-en-el-discurso-oral-de-estudiantes-e-le-universitarios-de-nivel-b1-en-contexto-de-inmersion (07/12/2015).

Medina, I. (2013). "Los elementos atenuadores para expresar desacuerdo en el discurso oral de estudiantes ele. Estudio cuantitativo", Marcoele: Revista de Didáctica, 16: http://marcoele.com/ descargas/16/medina-atenuadores.pdf (07/12/2015).

Meyer-Hermann, R. (1988). "Atenuación e intensificación (análisis pragmático de sus formas y funciones en el español hablado)", Anuario de Estudios Filológicos, 11, pp. 275-290. 
Peirce, B. N. (1995). "Social identity, investment, and language learning”, TESOL Quarterly, 29, pp. 9-31.

Robles, P. (2014). "Competencia plurilingüe y estrategias de atenuación en el cambio de código lingüístico de L1 a L2/L3”. Actas del II Congreso Internacional Nebrija en Lingüística Aplicada a la Enseñanza de Lenguas, Universidad Nebrija (26-28 de junio de 2014).

Searle, J. (1969). Actos de habla. Traducción L. M. Valdés. Madrid: Cátedra, 1980.

Schmidt, R. (1993). Consciousness, learning and interlanguage pragmatics. En Kasper, G. y S. BlumKulka (eds.), Interlanguage pragmatics. New York: Oxford University Press: pp. 21-42.

Vázquez, J. A. (2008). "Mecanismos de atenuación en español eitaliano: quizá y forse". RedELE, 12: http://www.mecd.gob.es/dctm/redele/MaterialRedEle/Revista/2008_12/2008_ redELE_12_08VazquezPerez.pdf?documentId=0901e72b80de14e7 (07/12/2015). 